\title{
Filigrane
}

Écoutes psychothérapiques

\section{D’un fond abyssal de la langue}

\section{Ghyslain Lévy}

Volume 20, numéro 1, printemps 2011

La langue vivante de la clinique psychanalytique

URI : https://id.erudit.org/iderudit/1004037ar

DOI : https://doi.org/10.7202/1004037ar

Aller au sommaire du numéro

Éditeur(s)

Revue Santé mentale au Québec

ISSN

1192-1412 (imprimé)

1911-4656 (numérique)

Découvrir la revue

Citer cet article

Lévy, G. (2011). D’un fond abyssal de la langue. Filigrane, 20(1), 23-32. https://doi.org/10.7202/1004037ar

\section{Résumé de l'article}

Comment l'expérience de la psychanalyse peut-elle rendre compte de ce qui fait la relation vivante de chacun à l'étrangeté de "sa » langue, en particulier quand celle-ci porte des parts d'indicible, de douleur innommable, de catastrophe subjective irreprésentable, hors de toute mémoire et de tout souvenir ? La confiance faite à l'autre est ici décisive, et constitue tout l'enjeu de l'analyse permanente des contre-transferts mobilisés en séance. En quoi la dominance actuelle d'une langue de la technique, réduite à son savoir-faire, en vient à disqualifier la fonction identifiante de la parole, au risque d'ouvrir à cette part refoulée de la langue qu'on pourrait désigner comme " post-totalitaire " ? Il s’agira ici de repérer ce qui, dans la clinique de la séance, renvoie à un tel drame de la langue, dans la destitution transmise de son pouvoir de nomination.
Ce document est protégé par la loi sur le droit d'auteur. L'utilisation des services d'Érudit (y compris la reproduction) est assujettie à sa politique d'utilisation que vous pouvez consulter en ligne.

https://apropos.erudit.org/fr/usagers/politique-dutilisation/ 


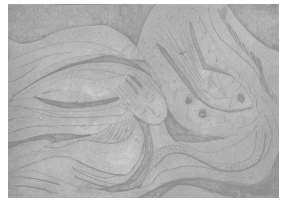

\title{
D'un fond abyssal de la langue
}

\section{Ghyslain Lévy}

\begin{abstract}
Comment l'expérience de la psychanalyse peut-elle rendre compte de ce qui fait la relation vivante de chacun à l'étrangeté de "sa" langue, en particulier quand celle-ci porte des parts d'indicible, de douleur innommable, de catastrophe subjective irreprésentable, hors de toute mémoire et de tout souvenir? La confiance faite à l'autre est ici décisive, et constitue tout I'enjeu de l'analyse permanente des contre-transferts mobilisés en séance. En quoi la dominance actuelle d'une langue de la technique, réduite à son savoir-faire, en vient à disqualifier la fonction identifiante de la parole, au risque d'ouvrir à cette part refoulée de la langue qu'on pourrait désigner comme "post-totalitaire" ? II s'agira ici de repérer ce qui, dans la clinique de la séance, renvoie à un tel drame de la langue, dans la destitution transmise de son pouvoir de nomination.
\end{abstract}

L'asservissement du langage dans le bavardage aboutit presque inévitablement à l'asservissement des choses dans la folie.

W. Benjamin. Sur le langage. Euvres I

nterroger ce qui fait le lieu vivant de la langue, sa condition vivante ou survivante, ce qui fait la relation vivante et paradoxale non au propre de la langue mais à son impropre, non à son appropriation mais à son ex-appropriation, revient nécessairement à me ramener à mon expérience de la psychanalyse, c'est-à-dire à la mise à l'épreuve singulière d'une telle interrogation. Comment parvenir à en rendre compte si ce n'est à partir d'une acuité de l'écoute qui vienne donner place pour la première fois à ce qu'une parole ne dit pas, à ce qui n'a même pas de place dans la langue elle-même, qui n'est figurable ni en termes de vide, d'absence, de faille ou de manque? À moins que, quant à ce que je cherche ici à approcher, il s'agisse plutôt de l'inverse: comme un excès, un trop de présence, une langue qui ne porterait qu'un trop plein de rage, de colère, de fureur, une langue figée dans l'immobilité, la paralysie, une sorte de glue, trop de matière qui empêche et entrave tout mouvement. Une parole engluée ou prise en masse dans la glace. Un paysage intérieur d'éboulis, de crevasses, de fractures rocheuses, une géographie interne ravinée qu'aucune parole n'est encore parvenue à féconder, à animer, à rendre vivante. Pas de douleur mais pas non plus de vie. Aucune trace qui fasse mémoire, souvenir, témoignage de ce qui est arrivé à la langue catastrophée. «Tel un gigantesque rêveur qui tente de se retourner, mais en vain ${ }^{1}{ }^{\star}$ la langue s'arrête sur le bord du glacier de la pensée, y découvrant l'abîme au-dessus duquel elle se tient.

Aujourd'hui la séance commence comme dans un rituel bien réglé, répétant les innombrables séances précédentes, un rituel de silence rythmé par un habituel «rien 
à dire » qui vient refermer aussitôt ce que la séance ouvrait de possibilité. Du côté de l'analyste, toujours le même rendez-vous avec le sentiment d'impuissance, d'échec, d'ennui, le même étonnement devant une résistance à ce point inébranlable. Soudain une évidence qui surgit brutalement, intolérable : l'immobilité de ce corps gisant sur le divan, et, du côté de l'analyste, la sensation violente d'être soi-même pris dans la même sidération pétrifiée... Deux gisants pétrifiés dans la mort, unis à jamais. Pour l'analyste, l'urgence à se sentir vivant, et à communiquer à son double silentiaire ce qui, du surgissement de cette scène, vient de le saisir. Et la surprise en entendant la voix qui monte du divan, le ton détaché et presque amusé avec lequel celle-ci s'étonne en constatant qu'il ne se passe rien, comme d'habitude. Ouverture brutale d'une perception extrêmement vive du côté de l'analyste, celle d'un clivage violemment actif qui vise à faire vivre à l'analyste une expérience terrifiante de pétrification et de terreur, en présence d'un autre qui en nierait la réalité et en banaliserait le ressenti. Quelqu'un terrorise quelqu'un d'autre et dénie en même temps la réalité de cette expérience de terreur. La langue ne vient-elle pas toujours interroger le fond catastrophique dont elle doit s'arracher, ce fond de silence ou plutôt de mutisme qui la guette, cette obscurité qui la menace? Autrement dit le rapport humain au langage se fait toujours sur fond d'asservissement dans le bavardage, la confusion des langues, la tristesse ou le mutisme. Comme l'écrit W. Benjamin : "Il est en toute tristesse un très profond penchant au mutisme, ce qui est infiniment plus qu'une impuissance ou qu'une aversion à communiquer. ${ }^{2}$ »

Freud, dès ses premiers écrits, en avait eu l'intuition en situant dans la chambre d'enfant le premier drame de la langue. Chambre obscure des angoisses primitives, celles de l'enfant dans le noir, cette chambre obscure des terreurs primaires, une chambre que nous portons tous en nous durant toute la vie. «Du moment que quelqu'un parle, il fait clair ${ }^{3}$.» Cet appel, Freud va le mettre dans la bouche de l'enfant pour vaincre sa terreur, appel à l'autre, à un autre qui ne serait plus l'étranger, l'hostile, le menaçant, le haï, mais appel à un autre dont la parole serait désormais capable d'éclairer soudain l'obscurité du monde. Une allumette qui craque dans le noir, ne serait-ce pas l'image d'une parole qui résiste aux ténèbres, et qui, de sa fragile lueur, tremblante, éphémère, fait reculer les terreurs de l'habitant de la chambre? La parole réalise l'avènement de sa figuration, de cette urgence à produire des pensées-images, des mots-lucioles selon la belle expression de Didi-Huberman ${ }^{4}$, flammèches illuminant soudain avant que de s'éteindre la nuit de nos terreurs privées et collectives, petites survivances tendues vers les «luciferi», nos porteurs de lumière internes. Toute demande d'analyse n'est-elle pas en fin de compte appel à «quelqu'un» qui rende à la parole cette fonction: voir clair ou du moins, comme le dit Freud, voir clairement les obscurités?

Toute la question de la confiance faite à l'autre, à sa fiabilité est ici en jeu. Et c'est tout l'enjeu contre-transférentiel qu'implique la fonction analytique, d'y entendre l'appel à ce « quelqu'un » dont la parole permettrait de voir dans l'obscur, comme de voir l'obscur. En chacun il y a un habitant de la chambre. En chacun aussi ce lieu de terreur sans nom, sans mot, ce point d'opacité et d'énigme, cet état de désolation et 
d'effroi, en attente d'une présence qui parle dans le noir. Je pense à ce propos à cette toute jeune fille qui évoquait le noir absolu de sa chambre comme étant aussi celui de sa tête. La tête comme une chambre sans porte ni fenêtre. Le noir absolu du vide, sans limites ni repère. Elle aussi, seul habitant d'une chambre-tête dont il serait impossible de sortir. Jusque-là aucun autre dont la parole ouvrirait une issue. Dans la nuit totale de la tête, l'habitant de la chambre ne perçoit que les mouvements de son propre corps et les battements de son cœur, seuls repères auxquels se raccrocher. De l'autre, de sa proximité, elle n'en perçoit que le vide vertigineux, l'abîme qui s'ouvre quand on saute à l'élastique. Alors comment sortir de sa propre tête? Longtemps seule la pensée de se tuer a pu représenter pour elle l'unique échappée à cette sphère noire qui aurait représenté sa chambre d'enfant. Elle avait ensuite imaginé qu'en disposant des posters fluorescents sur les murs, elle réussirait ainsi à créer des poches de lumière qui joueraient le rôle de fenêtres, à la façon de ses idéaux, la danse par exemple, qui auraient joué le même rôle dans sa vie.

Le noir n'est pas l'obscur. Le noir renvoie au trou, au défaut de toute représentation, au vide sans fond, sans limites, sans figure. L'obscur par contre est toujours gros de présences potentielles, relève de ce qui se promet tout en se cachant, de ce qui s'ouvre à l'énigmatique, au théâtre des jeux de l'ombre et des lueurs, à ses différentes scènes. Quand quelqu'un parle, le noir de la chambre d'enfant se transforme peu à peu en obscurité, si on peut dire. C'est là l'intuition freudienne qui place au début, au commencement de la psychanalyse, cet appel à une parole susceptible de transformer l'absolue détresse humaine, le noir dans lequel s'abolit tout sentiment d'exister, en une interrogation angoissée sur le mystère de l'être.

À l'autre bout de l'œuvre freudienne, au moment de son terme, on retrouve à nouveau la chambre d'enfant. En 1930, Freud la convoque encore, mais cette fois-ci ce n'est plus pour y faire intervenir cet autre dont la parole, appelée par l'habitant terrorisé dans le noir, fera la lumière. Avec le constat freudien d'un malaise dans la culture, le monde, par sa destructivité terrifiante, l'ivresse du pire qui en témoigne, à la fois individuellement et collectivement, devient la puissance de mort menaçant d'effraction les murs de la chambre d'enfant. «L'expérience nous l'enseigne: le monde n'est pas une chambre d'enfant. » L'œuvre freudienne est ainsi tendue entre deux scènes dont la chambre d'enfant occupe le centre, et dont le drame de la langue sera l'enjeu. En 1905 la terreur occupe l'intérieur de la chambre, y plongeant son habitant dans l'effroi de sa disparition dans le noir infini. L'issue provient de l'extérieur, ce quelqu'un qui parle et qui, en s'adressant à l'habitant de la chambre, va le faire exister. En 1930, les temps se sont assombris. Le noir n'est plus seulement dedans. Il s'est étendu à toute la réalité extérieure. La personne secourable qui, au début de la psychanalyse, portait l'espoir d'une parole qui repousse les limites de la néantisation, est désormais introuvable. L'œuvre freudienne s'achève sur un doute terrible quant à la capacité de la langue à ne pas sombrer elle-même dans le naufrage de la civilisation, tel qu'en fait le constat Le malaise dans la culture. Plus de chambre d'enfant dans laquelle trouver refuge, se replier pour y protéger un narcissisme de survie, pour y sauvegarder les restes linguistiques porteurs des images-lucioles faisant encore 
résistance. La constatation freudienne finale a quelque chose d'implacable: l'expérience nous l'enseigne, écrit Freud. C'est la force de l'expérience qui désormais fait force de loi. Plus d'illusion : la noirceur du monde a tout envahi. Et l'enfance, l'infantile inconscient n'ont plus droit de cité. Le monde n'est pas une chambre d'enfant, autrement dit le monde du rêve, de l'illusion, de l'enfance, c'est à dire aussi celui de l'infantile inconscient et donc la possibilité de la psychanalyse, sont menacés de voler en éclats avec la chambre. Le monde de la destruction règne en maître.

Le drame de la langue traverse précisément toute l'œuvre freudienne car si celleci avait, au début de la psychanalyse, le pouvoir de faire la clarté et de repousser au plus loin les ombres envahissantes, de sauver ainsi la subjectivité humaine en l'arrachant à l'indicible, c'est désormais, sous la souveraineté de la pulsion de mort, la langue elle-même qui devient paradoxalement l'instrument du meurtre de la subjectivité. J'ai eu l'occasion ailleurs de revenir sur la fonction meurtrière de la novlangue nazie, à partir des relevés systématiques que pouvait en faire Victor Klemperer. Toute forme collective génocidaire porte atteinte à l'organisation sémantique de la langue, en l'instrumentalisant comme appareil de mort. Cette pervertisation de la langue a trouvé dans le génocide rwandais de 1994 sa réactualisation exemplaire puisque, par les mots démétaphorisés de la langue commune, les bourreaux ont tué, et par les mots de la culture, ils ont nié l'existence des meurtres. Les proverbes ne furent-ils pas utilisés en les détournant de leur sens figuré pour être transformés en mises en scène de meurtres à actualiser? De même pour les tabous et les interdits portant sur l'inceste, le cannibalisme et le meurtre qui furent pris à la lettre pour les retourner en injonctions et en obligations, désacralisant la fonction sociale du tabou en y portant atteinte. Comme lors de tous les génocides, le sens des mots fut détourné dans la visée d'une banalisation et d'un déni de la réalité du meurtre. La langue des génocides est une langue technique, c'est-à-dire une langue souveraine par laquelle sont communiquées les technologies de la destruction et du meurtre de masse.

La question de la langue interrogée par la psychanalyse ne peut ainsi faire l'économie de ce qui constitue tout régime de parole dans un système démocratique, et de ce qui la destitue en instituant un régime de la langue qui porte l'héritage de sa déshumanisation post-totalitaire. En quoi peut-on dire qu'aujourd'hui, avec la souveraineté de la langue technique, les conditions post-totalitaires de la parole s'avèrent réunies pour en instituer l'ordre? Il s'agirait préalablement de s'interroger sur les effets de la parole, effets de sens et effets de force, en tant que toute parole est pourvue d'effets, c'est-à-dire aussi en tant qu'elle est parole adressée. Ce qui pose d'emblée la question du destinataire, et des effets sur ce destinataire. C'est bien là ce qui constitue les mots comme lestés d'un sens et d'une force pulsionnelle dont l'enjeu essentiel reste leur valeur de vérité pour le sujet qui les énoncent et les destinent. Valeur de vérité pour le sujet énonciateur et effets sur le destinataire constituent les deux conditions de la parole en régime démocratique. C'est aussi ce qui fait que la parole est action, fondamentalement. La langue s'organise sur une énonciation à laquelle le sujet s'identifie. Cette fonction identifiante de la parole pour le sujet qui l'énonce la constitue en valeur de vérité. 
En quoi le dérapage de cette fonction identifiante de la parole, du fait de la dominance de la langue technique, contribue aujourd'hui à un mode de déni organisateur d'une destitution de cette fonction? En quoi cette destitution de la fonction identifiante de la parole risque d'ouvrir sur ce que j'appelle le régime post-totalitaire de son fonctionnement? Plusieurs procédures sont ici en jeu qui produisent une paroleacte et la perte de sa fonction de référence pour un sujet visant à devenir une stricte manipulation de signes et de traits identifiants ou plutôt discriminants. La violence de cette parole-acte est bien d'une part de renoncer à toute référence susceptible de soutenir la cohérence entre le sujet énonciateur et l'énoncé lui-même. La parole-acte est un énoncé marqué par le déni: je dis que je vous aime, mais c'est une façon de vous interdire d'entendre que je vous hais. Autrement dit la parole-acte est une parole meurtrière de tout destinataire, au sens où elle est meurtrière de sa capacité de penser. Elle procède par un double système d'injonction paradoxale, comme Piera Aulagnier a pu le théoriser. Car le déni porte non seulement sur le contenu : je dis que je vous embrasse pour vous interdire d'entendre ma volonté de vous tuer. Mais le déni porte aussi sur l'organisation même du discours, puisque mon discours fait semblant de m'adresser à vous alors qu'en fait, il vous annule.

À cette parole-acte qui s'installe hors de toute référentialité dans l'usage par exemple de l'insulte prise au mot dans l'acte de meurtre, il faut ajouter une autre dimension du discours, toujours à l'adresse du destinataire: celle d'un déni de haine interdisant à l'autre tout moyen de donner sens à l'énoncé dans sa fonction d'insulte, et dans sa valeur performative de violence et de haine. L'injure ne peut être relevée puisqu'elle est aussitôt désavouée : elle ne serait que «mot d'enfant», «lapsus», «jeu de mot», "enfantillage», et c'est celui qui s'en offusque qui doit porter la charge de la responsabilité d'une haine qui lui est ainsi déléguée. Le rôle de la parole-acte est toujours de s'adresser à un destinataire isolé des autres, discriminé et séparé de la communauté des autres, un individu atomisé, comme l'écrit Hanna Arendt. C'est sur la solitude du sujet écarté de tous les autres que la parole-acte tente d'exercer son effet de massification, c'est-à-dire d'agglutination des sujets désubjectivés, déprivés de toute parole, réduits à des corps sans nom, et désignés par quelques traits disqualifiant qui font effet de foule.

Parmi ces traits que le discours post-totalitaire privilégie aujourd'hui, le sang constitue le lieu d'une condensation faisant intervenir sous le même signe négatif, l'autre comme l'étranger, le haï, l'hostile, le mauvais, et sa «transposition» biologique, le virus du VIH comme trace de l'impur, du corps étranger et de son pouvoir de contamination, de franchissement des frontières, barrières et limites.

Le sang, mais aussi le Nom propre, sont aujourd'hui les lieux où la question même de la différence se trouve portée à sa pointe extrême, à son point le plus difficile à penser, là où l'identité vient faire, à l'épreuve de l'autre, l'expérience vacillante de son incertitude, ou plus précisément de son indécidabilité. Indécidable comme l'est fondamentalement la question même de l'origine, et ici l'injure portant sur le Nom propre, ou le doute sur le sang, renvoient projectivement à l'autre la charge d'en soutenir et l'opprobre et son épreuve. Indécidable comme l'est tout autant le 
nouage du sexe et du meurtre, tel que, autour de la menace réelle de contamination par le virus VIH, s'est condensée leur double représentation.

Quant aux conditions héritées aujourd'hui du régime totalitaire de parole dans la langue, elles ne sont efficaces qu'articulées à un exercice de violence sur les mémoires, sous forme d'un après-coup paradoxal. Autrement dit, et contrairement à l'après-coup perlaboratif qui procède en donnant sens traumatique rétroactivement à un événement initial «insignifiant », à partir de l'effet rétroactif d'un fantasme ou d'un autre événement, l'après-coup paradoxal vient annuler rétroactivement l'histoire, et en réécrire après-coup une version ad hoc, conforme aux circonstances de l'actuel. Ainsi l'organisation d'un après-coup paradoxal vise-t-il l'effacement du sens plutôt que son émergence, voire même la construction d'un sens « officiel » imposé, surimposé, falsifié.

Ceci dit les actes psychiques d'annulation rétroactive et d'après-coup paradoxal visent non seulement l'effacement des traces mnésiques au profit d'une "mémoire officielle ", mais visent en même temps l'effacement de l'acte même d'effacement, selon la technique de l'injonction post-hypnotique et de son effacement par une surinjonction imposant l'oubli de la première injonction. Freud le soulignait dans L'homme Moïse et le monothéisme: «Il ne suffit pas de commettre un meurtre, fautil encore en effacer les traces. » Ceci reste vrai dans la langue, au-delà de tout système politique collectif, dans tout système familial ou communautaire qui rend fou.

Ainsi le régime post-totalitaire de la parole procède-t-il par la destitution de la fonction de représentance de la langue, rendant définitivement inaccessibles les traces mnésiques refoulées, si ce n'est sous la forme d'un passage au somatique, et condamnant à la stricte répétition dans l'actuel de tout événement hors psyché. N'est-ce pas d'ailleurs là l'étrange paradoxe qui contraint ceux qui se donnent pour tâche de «réviser» l'Histoire en en effaçant les traces, que de s'installer dans la répétition même de l'événement qu'il s'agit de nier? Le déni ne s'organise-t-il pas toujours sur la contrainte à répéter?

Pour faire un pas de plus, il faudrait préciser que l'injonction qui interdit le souvenir n'est pas sans affinité avec l'autre injonction, apparemment contradictoire, celle qui interdit d'oublier, à la différence considérable cependant que la première ne se contente pas d'interdire l'accès au souvenir, elle procède par falsification de la mémoire, fausse remémoration, et après-coup paradoxal. Plutôt que de parler d'interdit d'oublier, il serait peut-être plus juste de dire qu'il s'agit d'un interdit de s'en remettre, de se dégager de cette culpabilité propre au survivant qui, arrêtant la vie à l'instant de l'événement, ne peut organiser qu'un culte définitif de la mort ou des morts, sur lequel se fera la transmission aux générations suivantes.

La psychanalyse a également à se confronter à une telle violence d'effacement active dans la langue et pouvant porter sur un déni de réalité quant à la représentation de la réalité perceptive. Ici le régime post-totalitaire de la parole réside en une parole actante, ou plutôt active quant à sa capacité de détruire certaines pensées, de volatiliser la pensée ou de la réduire à des automatismes programmés. Comme dans la psychose « où le refoulé est décidé par un autre et répond à un ordre tout à fait 
arbitraire $^{5}$ ", l'acte d'effacement commis dans la langue vient répéter la catastrophe psychique, privée ou collective, catastrophe déjà subie, en en interdisant toute possible re-présentation. Car c'est l'activité de représentation qui se trouve désinvestie, témoignant dans ce registre de la prévalence des pulsions de mort. Il est désormais interdit de savoir, de comprendre, de se souvenir. Les pulsions épistémophiliques et scopiques peuvent même se mettre au service des techniques de meurtre, ainsi qu'on a pu l'observer récemment lors du génocide rwandais. Comme dans le registre de la psychose, il en est de même dans des situations collectives de meurtre de masse : il est interdit de penser l'interdit. Toute langue totalitaire, privée ou collective, s'organise autour d'une activité de déni qui vient en lieu et place d'un impossible refoulement. Parole porteuse de ce non-refoulé dénié et violemment actif envers un autre qui, lui, serait chargé d'incarner, mais à quel prix, l'instance refoulante manquante ou défaillante chez l'énonçant. Par exemple l'enfant tenu d'incarner en son insu cette instance refoulante défaillante en la psyché maternelle. Simultanément s'opère, dans la langue maternelle, l'effacement de cette injonction psychique elle-même, l'effacement de toute possible mémoire d'une telle délégation d'instance d'une psyché à l'autre, soit la mort psychique du destinataire.

Dans la clinique de la séance, un tel drame de la langue peut trouver à se repérer par l'effacement au fur et à mesure des interprétations étayant le processus de subjectivation en cours. Ceci témoigne d'une mise en acte inconsciente des pulsions de mort œuvrant contre l'activité psychique de mémorisation et contre l'investissement des traces mnésiques afférentes au travail analytique de perlaboration.

D'où l'importance, à partir d'une telle expérience de la langue que la clinique analytique permet d'éclairer, d'interroger la langue vivante de la psychanalyse à l'aune de son pouvoir de nomination. Un texte de W. Benjamin que j'ai utilisé précédemment, Sur le langage ${ }^{6}$, me servira ici de détour pour interroger autrement ce qu'il en est du langage humain, et la façon dont la situation analytique le met en scène. Le langage humain nomme, et dans cet acte de nomination, il se communique, c'est-à-dire qu'il communique ce que W. Benjamin appelle « son essence spirituelle», ce que nous désignons aujourd'hui dans notre jargon par le terme de subjectivation ou de subjectivité. C'est ce pouvoir de se communiquer qui, pour W. Benjamin, singularise le langage humain. Cette dimension spirituelle du langage humain se traduirait non par l'acte de nomination, mais dans l'acte de nomination. Je l'entends comme présence d'énigme en chaque mot, présence ou puissance de l'énigme qui habite en chaque mot. Freud ne le disait-il pas lui-même en termes d'inconscient pulsionnel doublant l'acte de parole? Autre façon de dire ce qu'écrit W. Benjamin: "L'essence spirituelle se communique dans un langage et non par lui. » Autre façon aussi de distinguer le langage humain en tant qu'il offre hospitalité à son " essence spirituelle», laquelle se communique en lui, et d'autre part, ce qu'il en est d'un langage technique qui, lui, aurait renoncé à tout pouvoir de nomination. Le langage technique aurait perdu avec le nom la puissance d'énigme qui habite le mot. Le langage technique, contrairement au langage humain, ne s'adresse pas. Il communique des ensembles de signes, il ne se communique pas. Il aurait refoulé l'abîme que constitue en chaque 
mot son « essence spirituelle», c'est-à-dire sa subjectivité. D’où la parole-acte et son potentiel meurtrier que véhicule tout langage technique, c'est-à-dire non-humain. Le langage technique ne nomme pas, on pourrait même dire qu'il dé-nomme. D’où cette remarque si juste de Jacques Rancière à propos de la surcharge d'images qui envahissent notre champ perceptif, des images qui sont saturées de corps sans nom, «trop de corps qui sont objets de parole sans avoir eux-mêmes la parole ${ }^{7}$ ».

Qui dit abîme dit aussi expérience de bord, du bord de l'abîme. C'est précisément cette expérience de bord qui se communique dans le langage humain, et qui exprime sa force spirituelle, soit le contraire même d'un langage technique qui lui, se contente de communiquer. On pourrait dire que l'homme communique par le langage technique, mais il ne se communique pas dans lui. C'est peut-être là que peut s'approcher ce qu'il en est de l'expérience vivante de la langue dans la situation analytique comme expérience humaine, c'est-à-dire dans laquelle l'homme se communique. La langue et les noms sont abyssaux, et c'est de ne pas le comprendre qu'on risque de tomber dans l'abîme. L'expérience de la psychanalyse est à ce titre aujourd'hui décisive puisque nous confrontant à ce dilemme radical: dans l'acte transférentiel de parole, dans l'acte d'énonciation interprétatif, dans leur fonction même de nommer, se réalisent des actes performatifs de langage, comme je l'ai rappelé à propos de la chambre d'enfant soudain éclairée « quand quelqu'un parle». La psychanalyse est une situation spécifique en donnant au sujet la possibilité de se nommer, de se communiquer dans les mots abyssaux du langage humain, d'y loger ce que W. Benjamin appelle sa force spirituelle. Peut-être pourrions-nous aussi l'appeler la force du sacré? Bien sûr faudrait-il aussitôt que nous arrêtions sur le sens de ce sacré dont Freud dénonçait la source religieuse pour mieux le reconduire sous la forme de sa croyance dans la science comme religion du Progrès.

La psychanalyse n'aurait-elle rien à dire du sacré? Certes Freud n'a ni réhabilité le sacré, ni cherché à sacraliser le sens, mais il a cherché un sens au sacré, en montrant que celui-ci n'était ni un délire pur ni une mystique de la pureté, mais la forme dramatique que prenait nécessairement la conflictualité propre à la vie psychique. Freud a bien désacralisé le sacré en le ramenant à sa dimension essentielle, celle du tragique auquel la condition humaine confronte chaque sujet, avec l'énigme de son origine, de son ultime, avec cette puissance de désordre d'un sexuel auquel le sujet se trouve radicalement aliéné.

Ceci dit, il y a une affinité entre cette force spirituelle qui fait le langage humain et la force de sacralité de la langue, un sacré qui, évidemment, n'est à rabattre ni sur le religieux ni sur le divin. Il y a une sacralité de la langue en tant qu'elle est «tenue au secret » comme l'écrit J. Derrida, tenue par le secret, à entendre comme le lieu d'une ouverture infinie, sans limite de la vie psychique. Freud ne le disait-il pas à sa façon : "Psyché est étendue, ne le sait pas. » La sacralité du langage humain tient précisément de cette psyché qui ne sait pas, c'est-à-dire d'un inaccessible auquel l'homme est à jamais soumis, d'un mystère de l'être qui, avec le secret, se communique dans la langue. N'est-ce pas ce que rappelle la psychanalyse quand elle situe les premières expériences du petit humain dans l'épreuve sans langage de la perte de 
l'Autre, de son manque, de son défaut, et donc dans l'hallucination du désir de sa présence? N'y a-t-il pas avec l'expérience analytique et sa pratique singulière de la langue comme lieu d'accueil de l'inconscient, une ouverture à ce qui s'inscrit dans la langue elle-même d'un refoulement originaire, d'un effacement premier, d'un oubli avant toute mémoire, d'un retirement au fondement de toute pensée? Le secret, dans l'affinité qui le lie à la sacralité de la langue, est désir de secret, certainement pas contenu positif mis au secret. Ne renvoie-t-il pas toujours au sans-langage qui en serait la pointe abyssale, et que le désir de secret recouvrirait de son voile? Pour reprendre les mots de Blanchot, toujours «le piétinement de cet inconnu sans langage, là à notre porte, sur le seuil.»

Le désir de secret serait la condition d'un tel retirement originaire qui, dans le langage humain, se communique, et en constitue le fond abyssal. M. Blanchot écrit à ce propos: « Retirement et non développement. Tel serait l'art, à la manière du Dieu d'Isaac Louria qui ne crée qu'en s'excluant ${ }^{8}$.» Créer en s'excluant. Telle serait une autre façon d'approcher cette force spirituelle que W. Benjamin appelle le langage humain: se communiquer en passerait par le mouvement de "se retirer", de s'exclure de l'acte même de créer, et ainsi de soutenir dans sa propre parole la position de sujet. Peut-être que «l'étendue» de la psychanalyse, l'étendue psychique de son acte, n'est rendue possible qu'au prix d'un tel retirement auquel répond le retrait propre à la fonction analytique.

Tout autre chose est cette transparence sans secret propre au langage technique auquel notre civilisation techno-scientifique donne aujourd'hui priorité, dans l'exhibition d'un «tout-montrer» qui écrase toute profondeur de la langue, abolit son étendue et son obscurité, dénie le fond abyssal qui en constitue l'énigme. En quelque sorte il y aurait avec cet écrasement du langage technique l'équivalent d'un meurtre de la langue dans sa fonction de nomination, d'appel, dans cette fonction d'abri linguistique que la chambre d'enfant vient représenter pour chacun.

Comment une langue qui a perdu son pouvoir de nomination peut-elle se transmettre? L'instrumentalisation technique de la langue, son effet dans l'écrasement des mots, de leur épaisseur, par leur réduction sémantique, a créé ce que J. Derrida appelle « une platitude» de la langue, la production d'une langue de surface, superficielle, d'une langue à la surface de laquelle nous marchons aujourd'hui, tels des somnambules, ou du moins sur laquelle nous croyons marcher ${ }^{9}$.

La novlangue technologique à laquelle nous croyons est notre déni linguistique actuel dont l'effet est de décapiter la langue, comme le suggère Derrida ${ }^{10}$. C'est cette langue décapitée de sa force spirituelle, de sa puissance d'énigme, de son épaisseur abyssale, qui va se transmettre ainsi, dans le plus complet déni de son meurtre, aux générations suivantes. Or ce qui fait le langage humain est toujours ce que j'appellerais son esprit de retour, le mouvement de retournement par lequel le langage se retourne sur lui-même, et dans ce sens se communique, comme dit W. Benjamin. Le langage humain se communique dans ce retour sur soi, sur ce qui a été oublié, refoulé, effacé, tué, radicalement intransmissible. C'est donc une langue hantée, habitée de ses fantômes, qui est toujours en arrière de notre langue véhiculaire et instrumentalisée 
par la technique, de cette non-langue que nous transmettons aujourd'hui. Comme l'écrit M. Blanchot ${ }^{11}$, il s'agit là « d'un langage pétrifié auquel nous avons affaire, par lequel ne peut même plus se transmettre qu'il y aurait de l'intransmissible».

Il n'y a pas d'expérience de pensée, d'écriture ou de parole, qui ne soit une rencontre avec un échec à se reconnaître, l'échec d'une torsion sur soi-même, sur la trace de ce qui n'a pas eu lieu. Et à cet égard la situation analytique en serait d'ailleurs le paradigme. En d'autres termes il existe toujours un reste à dire, à écrire, qui a précédé toute tentative de dire ou d'écrire, une ruine au commencement de tout geste d'écriture, de tout acte de parole, de toute pensée qui se tord sur elle-même pour penser sa propre torsion, un échec inaugural à se reconnaître dans ce retour de la langue sur son fond abyssal. Ne serait-ce pas l'intransmissible qu'il y aurait lieu de transmettre?

Pour paraphraser M. Blanchot, parler?... Parler, n'est-ce pas toujours perdre le fil ? Mais peut-être aussi parfois écrire peut nous laisser dans cette discontinuité vertigineuse, quand le fil est devenu introuvable... Moment pour laisser le lecteur poursuivre à son tour...

Ghyslain Lévy

4, rue Dante, Paris 75005 ghyslainlevy@orange.fr

\section{Notes}

1. P. Lévi, À une heure incertaine, 15 mars 1946, p. 28.

2. W. Benjamin, Euvres I, Gallimard, coll. «Folio essais», 2000, p. 163.

3. S. Freud, Trois essais sur la théorie sexuelle, Gallimard, 1987, p. 168.

4. G. Didi-Huberman, Survivance des lucioles, Minuit, 2008.

5. P. Aulagnier, L'apprenti-historien et le maitre-sorcier, PUF, 1984, p. 259.

6. W. Benjamin, op. cit.

7. J. Rancière, Le spectateur émancipé, Éditions La Fabrique, 2008, p. 104.

8. M. Blanchot, L'écriture du désastre, Gallimard, 1980, p. 27.

9. J. Derrida, Les yeux de la langue, L'Herne, 2005, p. 39.

10. Ibid.

11. M. Blanchot, L'écriture du désastre, Gallimard, 1980, p. 207.

\section{Références}

AULAGNIER, P., 1984, L'apprenti-historien et le maître-sorcier, PUF, Paris. BENJAMIN, W., 2000, Euvres I, Gallimard, coll. « Folio essais ». BLANCHOT, M., 1980, L'écriture du désastre, Gallimard. DERRIDA, J., 2005, Les yeux de la langue, L'Herne. DIDI-HUBERMAN, G., 2008, Survivance des lucioles, Minuit. FREUD, S., 1987, Trois essais sur la théorie sexuelle, Gallimard. PRIMO, L., 1946, À une heure incertaine, 15 mars 1946.

RANCIÈRE, J., 2008, Le spectateur émancipé, Éd. La Fabrique. 